\title{
Isolated Polyarteritis Nodosa Presenting as Acute Epididymo-Orchitis: A Case Report
}

\author{
Christine Chhakchhuak ${ }^{\mathrm{a}, \mathrm{c}}$, Nishant Gupta ${ }^{\mathrm{a}}$, Elizabeth Turner $^{\mathrm{a}}$, Ronald Oxenhandler ${ }^{\mathrm{b}}$
}

\begin{abstract}
Polyarteritis Nodosa is a systemic necrotizing vasculitis typically affecting the small and medium sized blood vessels. It typically involves multiple organ systems with a wide variety of clinical presentations. Rarely, it affects only one organ of the body, also known as Isolated Polyarteritis Nodosa. Testicular involvement, although not uncommon with this disease, is rarely the first presenting sign. We present a case of a 62-year-old Caucasian male who presented with three-month history of pain, swelling and redness around the left testicular region. He was diagnosed with acute epididymoorchitis and started on broad spectrum antibiotics. However, with progressive symptoms, the patient finally underwent left orchiectomy. Histological analysis of the excised testicle revealed features consistent with microscopic polyarteritis nodosa. The patient was subsequently started on high dose steroids resulting in resolution of his symptoms. Thus, clinicians should consider polyarteritis nodosa in cases simulating a clinical picture of acute epididymo-orchitis.
\end{abstract}

Keywords: Polyarteritis Nodosa; Vasculitis; Epididymitis; Orchitis; Pathology

\section{Introduction}

Polyarteritis Nodosa (PAN) is a systemic necrotizing vasculitis typically affecting the small and medium sized blood vessels. The usual manifestations of this disease are protean and may involve multiple organs including skin, peripheral

\footnotetext{
Manuscript accepted for publication November 18, 2010

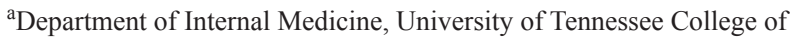
Medicine, Chattanooga, TN 37403, USA

${ }^{b}$ Department of Pathology, Memorial Health Care System \& Vanderbilt University, USA

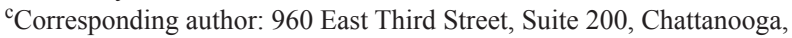
TN 37403, USA. Email: chris_chkchk@yahoo.co.in
}

doi: $10.4021 / \mathrm{jmc} 97 \mathrm{w}$ and central nervous systems, kidneys, joints, gastrointestinal tract, and heart. Nonspecific constitutional symptoms such as fever, fatigue, and weight loss are commonly seen as well [1]. On rare occasions, PAN may affect only one organ of the body, also referred to as isolated PAN [2]. On most occasions, isolated PAN can be treated with surgical excision of the affected organ or with a short course of systemic corticosteroids [3]. We describe a case of PAN simulating a clinical picture of acute febrile epididymo-orchitis with resolution of symptoms following orchiectomy and systemic corticosteroids.

\section{Case Report}

A 62-year-old Caucasian male presented to the hospital with a three-month history of pain, swelling and redness around the left testicular region. Prior to coming to the hospital, he had been seen by his primary care provider on three different occasions. He was initially diagnosed with orchitis and then with epididymitis. He had finished two courses of outpatient antibiotic treatments with levofloxacin and amoxcillin with no relief in symptoms. A month prior to his hospitalization, he was started on prednisone $40 \mathrm{mg}$ daily. One week prior, he was started on a tapering dose of prednisone and his symptoms flared back again which made him come to the hospital. His past medical history was significant for a diagnosis of chronic obstructive pulmonary disease (COPD) and gastritis. His past surgical history was significant for right middle lobectomy for bronchiectasis. The patient had a 50 pack year smoking history but had quit a few months prior to hospitalization. There was no history of alcohol or illicit drug use. His home medications included albuterol inhaler and famotidine taken on an as needed basis. On examination, the patient was afebrile and normotensive. A complete physical examination was unremarkable except for the left scrotal area which was warm, erythematous, and exquisitely tender to palpation. Significant laboratory data included an elevated ESR at 85 and an elevated C-Reactive protein (CRP) at 10.5. All other laboratory data including complete blood count, liver and renal function tests were within normal limits. A scrotal ultrasound was obtained which revealed a shrunken left testes, 
normal blood flow through the testes, and increased blood flow in epididymis consistent with epididymitis. The patient was started on broad spectrum antibiotic coverage with vancomycin and piperacillin-tazobactam. However, the patient continued to have progressive testicular pain and swelling. A urology consult was obtained and left orchiectomy was performed. Pathology analysis of the excised testicle and adnexa showed fibrinoid necrosis of medium and small sized arteries associated with PMN's, a brisk lymphoid infiltrate and occasional eosinophils and plasma cells. The angiocentric and destructive inflammation was present within the testicle, epididymis, and the loose connective tissue around the vas deferens. No thrombi or gross infarcts were seen (Fig. 1). Additional laboratory studies including antinuclear antibodies (ANA), rheumatoid factor (RF), serum complement levels, Venereal Disease Research Laboratory test (VDRL), hepatitis B surface antigen (HBsAg), hepatitis C antibodies, cryoglobulins, perinuclear and cytoplasmic ANCA, and HIV were negative. The patient was started on high dose corticosteroids with resolution in his symptoms.

\section{Discussion}

Polyarteritis Nodosa, as a systemic necrotizing vasculitis affecting medium and small sized blood vessels, was first described by Kussmaul and Maier in 1866. The extent of testicular involvement seen in PAN is reported to be between $38 \%-86 \%$, however, clinical symptoms are noted in approximately $18 \%$ of the patients with systemic PAN [4]. On rare occasions, systemic PAN presents with testicular involvement as the first sign [5]. Rare case reports of isolated testicular PAN have been described [3-8]. However, almost all of these patients had complete remission after surgical removal of the affected testicle with no requirement for either corticosteroids or immunosuppressive therapy. In the case report by Warfield et al, the patient had a relapse of his underlying

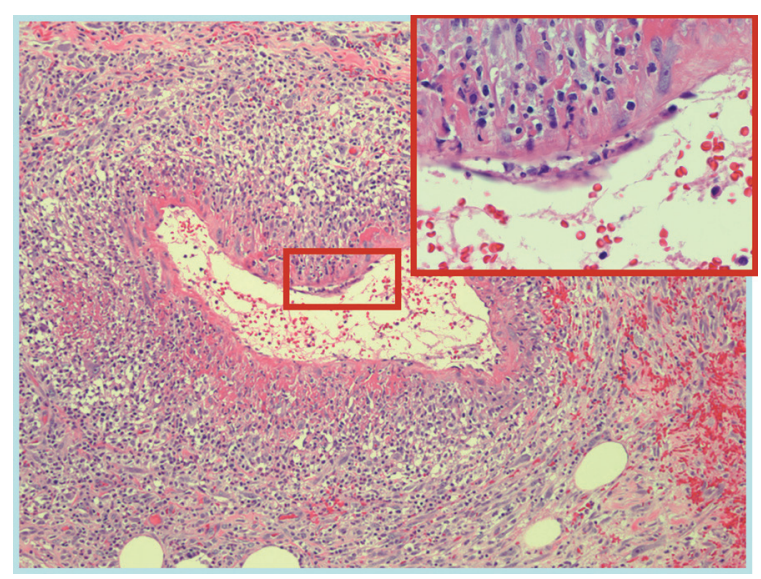

Figure 1. Histological specimen of the testicle showing necrotizing vasculitis with fibrinoid necrosis. disease in the contralateral testicle and he required long term therapy with steroids to achieve remission [8].

The exact etiopathogenesis of PAN is unknown. Isolated PAN of the testes may occur concomitantly with other connective tissue disorders [9]. Local immunostimulation following exposure to antigens or infectious agents may contribute to the development of isolated PAN [2].

The clinical presentation of testicular PAN is variable. It may be completely asymptomatic or present with features of pain, swelling and/or a local mass in some cases. This clinical presentation frequently leads to a misdiagnosis of acute orchitis, torsion, or neoplasm [4-8]. Clinical presentation of isolated testicular PAN is similar to systemic PAN with testicular involvement. Laboratory data usually is non-specific. Histological analysis of biopsy specimens remains the mainstay of diagnosis. The histological analysis of testicular PAN is also similar in both forms, however, the existence of infarcts in the affected testis is seen more often with systemic PAN [9]. Because it is unlikely to have systemic progression without evidence of underlying connective tissue disorder or cryoglobulinemia, a serological profile to include ANA, complement levels, ANCA, and Hepatitis panel was recommended within the pathology report and was negative [10].

Whether our patient had isolated PAN of the testis or systemic PAN with initial testicular involvement cannot be determined at this moment. He, however, would require close monitoring with special attention to the development of any other symptoms suggestive of a vasculitic process.

\section{References}

1. Sato, O, Conn, DL. Polyarteritis and microscopic polyangiitis. In: Rheumatology, Klippel, JH, Dieppe, PA (Eds), Mosby, St Louis, 2003.

2. Womack C, Ansell ID. Isolated arteritis of the epididymis. J Clin Pathol 1985;38(7):797-800.

3. Persellin ST, Menke DM. Isolated polyarteritis nodosa of the male reproductive system. J Rheumatol 1992;19(6):985-988.

4. Teichman JM, Mattrey RF, Demby AM, Schmidt JD. Polyarteritis nodosa presenting as acute orchitis: a case report and review of the literature. J Urol 1993;149(5):1139-1140.

5. Lee LM, Moloney PJ, Wong HC, Magil AB, McLoughlin MG. Testicular pain: an unusual presentation of polyarteritis nodosa. J Urol 1983;129(6):1243-1244.

6. Mukamel E, Abarbanel J, Savion M, Konichezky M, Yachia D, Auslaender L. Testicular mass as a presenting symptom of isolated polyarteritis nodosa. Am J Clin Pathol 1995;103(2):215-217.

7. Huisman TK, Collins WT, Jr., Voulgarakis GR. Polyarteritis nodosa masquerading as a primary testicular neoplasm: a case report and review of the literature. J Urol 
1990;144(5):1236-1238.

8. Warfield AT, Lee SJ, Phillips SM, Pall AA. Isolated testicular vasculitis mimicking a testicular neoplasm. J Clin Pathol 1994;47(12):1121-1123.

9. Shurbaji MS, Epstein JI. Testicular vasculitis: implica- tions for systemic disease. Hum Pathol 1988;19(2):186189.

10. Burke AP, Sobin LH, Virmani R. Localized vasculitis of the gastrointestinal tract. Am J Surg Pathol $1995 ; 19(3): 338-349$. 